\title{
Vitellogenin receptor selectively endocytoses female- specific and highly-expressed hemolymph proteins in the silkworm Bombyx mori
}

\begin{tabular}{|c|c|}
\hline Journal: & Biochemistry and Cell Biology \\
\hline Manuscript ID & bcb-2016-0255.R2 \\
\hline Manuscript Type: & Article \\
\hline Date Submitted by the Author: & 27-Mar-2017 \\
\hline Complete List of Authors: & $\begin{array}{l}\text { Han, Chaoshan ; Southwest University } \\
\text { Chen, Enxiang ; Southwest University } \\
\text { Shen, Guanwang; Southwest University } \\
\text { Zhixin, Peng; Southwest University, } \\
\text { Xu, Yinying; Southwest University } \\
\text { Zhang, Haiyan ; Southwest University } \\
\text { Liu, Hongling; Southwest University } \\
\text { Zhang, Yandi ; Southwest University } \\
\text { Wu, Jinxin ; Southwest University } \\
\text { Lin, Ying; Southwest University, State Key Laboratory of Silkworm Genome } \\
\text { Biology } \\
\text { Xia, Qingyou ; Southwest University }\end{array}$ \\
\hline $\begin{array}{l}\text { Please Select from this Special } \\
\text { Issues list if applicable: }\end{array}$ & N/A \\
\hline Keyword: & endocytosis, vitellogenin receptor, vitellogenin, storage protein 1 , silkworm \\
\hline
\end{tabular}


1 Vitellogenin receptor selectively endocytoses female-specific and highly-expressed

2 hemolymph proteins in the silkworm Bombyx mori

3 Chaoshan Han, Enxiang Chen, Guanwang Shen, Zhixin Peng, Yinying Xu, Haiyan Zhang,

4 Hongling Liu, Yandi Zhang, Jinxin Wu, Ying Lin, Qingyou Xia

5

6 State Key Laboratory of Silkworm Genome Biology, Southwest University, Chongqing,

7 China

$8 \quad *$.Correspondence: Ying Lin

9 Department of State Key Laboratory of Silkworm Genome Biology, Southwest University

10 Beibei District, Chongqing, 400716, China.

11 Phone: $29+86-23-68251953$;

12 Fax:+86-23-68251128.

13 E-mail: ylin197266@163.com 
Abstract

Vitellogenin receptor (VgR), a member of the low-density lipoprotein receptor (LDLR) family, functions to transport vitellogenin into the ovaries to protome ovarian growth and embryonic development. In insects, the only widely accepted ligand of $\mathrm{VgR}$ is $\mathrm{Vg}$. Recently, Bombyx mori $\operatorname{VgR}(\mathrm{BmVgR})$ has been shown to interact with Bombyx mori storage protein BmSP1 into certain cells. Although BmVgR could combine with Bombyx mori $V g$ (BmVg) and BmSP1, BmVgR did not affect the amount of BmSP1 taken up by Sf9 cells. Parallel immunofluorescence showed that most $\mathrm{BmVg}$ and $\mathrm{BmVgR}$ were localized in the inner oocyte membrane, showing tissue localization similar to that of $\mathrm{BmVg}$ labeled with pHrodo Red absorbed by the ovaries on day 2 of pupation. Although BmSP1 showed localization similar

to $\mathrm{BmVgR}$ during the same phase, little BmSP1 was present in the ovary. Additionally, BmSP1 did not exist in ovaries when the ovaries contained $\mathrm{BmVgR}$ on day 5 of pupation, suggesting that BmSP1 in the ovaries was not endocytosed by BmVgR. In summary, BmVgR could facilitate uptake of BmVg by developing oocytes, but did not modulate in the transport of BmSP1.

Key words: endocytosis; vitellogenin receptor; vitellogenin; storage protein 1; silkworm 
42

43

44

45

46

47

48

49

50

51

52

53

54

55

56

57

58

59

60

\section{Abbreviations}

BmSP1, storage protein 1 of Bombyx mori; BmVgR, VgR of Bombyx mori; BmVgR', the complete extracellular domain of BmVgR; BmVg, vitellogenin of Bombyx mori; EGFP, epidermal growth factor precursor homology domain; LBD, ligand-binding domain; LDLR, low-density lipoprotein receptor; $\mathbf{V g R}$, vitellogenin receptor; YPR, yolk protein receptor. 


\section{Introduction}

The vitellogenin receptor $(\mathrm{VgR})$ mediates the endocytosis of vitellogenin $(\mathrm{Vg})$ from the hemolymph to developing oocytes (Sappington and Raikhel 1998; Snigirevskaya et al. 1997). $\mathrm{VgR}$ is mainly located at the oocyte membrane surface of almost all oviparous organisms (Goldstein et al. 1985). Disruption of the function of $\mathrm{VgR}$ by natural mutations or genetic interference results in the phenotypes of "restricted-ovulator" (Schjeide et al. 1976) and yolkless (Ciudad et al. 2006; Schonbaum et al. 1995).

The VgR belongs to the low-density lipoprotein receptor (LDLR) family, and the ligands of LDLR family have been extensively studied. The LDLR family can combine with diverse the ovaries, such as the hypopharyngeal glands (Amdam et al. 2003), and fat bodies (Lin et al. 2015), suggesting that insect VgRs may also recognize multiple ligands. 
The domestic silkworm, Bombyx mori $(\mathrm{Bm})$ has been used as a Lepidoptera model organism. The full-length cDNA sequence of BmVgR has been obtained and BmVgR has been expressed specifically in the ovaries of silkworm. In silkworm with a partial deletion in the first epidermal growth factor precursor homology domain (EGFP) of BmVgR, the resulting eggs are whiter and smaller than normal eggs, and the homozygote is embryonic lethal (Lin et al. 2013).

The silkworm hemolymph circulatory system differs from that of higher animals in that it is an "open" system, bathing the internal organs including the ovaries. As a female-specific protein, $\mathrm{BmVg}$ is absorbed by the ovaries during pupation. Another female-specific protein Bombyx mori storage protein 1 (BmSP1) (Sakurai et al. 1988), is mainly synthesized in the fat bodies of female silkworms during the larval stage, secreting into the hemolymph and expressed abundantly in the hemolymph on the last day of larval stage. Most BmSP1 is then assimilated by the fat body again. However, few studies have attempted to identify or characterize the BmSP1 receptor. A recent study showed that LBD1 of BmVgR can specifically bind to the BmSP1(Liu et al. 2016).

Accordingly, in this study, we used several methods to confirm that the BmVg was endocytosed by $\mathrm{BmVgR}$ in silkworms. Additionally, using integrated methods verified in silkworms, we aimed to clarify whether BmVgR could participate in the transport of BmSP1.

\section{Materials and Methods}

\subsection{Cell-expression vector construction and expression}

We obtained full-length BmVgR from the carrier p50T6kb-9(stored in our laboratory). 
105 The forward and reverise primers for BmVgR (NM_001197251.1) were as follows: 106 5'-GAagatctTCATGAAGGTAGTTTTGTTAGCAATAGTTCTATGT-3' and 5'-TTGCGGCCGC 107 TTAATTGAGAAATTTATTTTT-3'. BmVgR' is the complete extracellular domain of $108 \mathrm{BmVgR}$ and does not affect the interaction with extracellular ligand; thus $\mathrm{BmVgR}$ ' is a 109 secreted protein. The primers for $\mathrm{BmVgR}^{\prime}$ were as follows: 110 5'-GAagatctATCAGAATGAAGGTAGTTTGTTA-3' and 111 5'-ATAAGAATgcggccgcTTAAGCGTAGTCTGGGACGTCGTATGGGTAGCAGACACAC 112 ACCGGAGC-3'. Polymerase chain reaction (PCR) was carried out at $96{ }^{\circ} \mathrm{C}$ for $15 \mathrm{~min}$; 113 followed by 5 cycles of $94^{\circ} \mathrm{C}$ for $40 \mathrm{~s}, 50^{\circ} \mathrm{C}$ for $45 \mathrm{~s}$, and $72^{\circ} \mathrm{C}$ for $5 \mathrm{~min} 40 \mathrm{~s}$; an additional 11430 cycles of $94^{\circ} \mathrm{C}$ for $40 \mathrm{~s}, 60^{\circ} \mathrm{C}$ for $45 \mathrm{~s}$ and $72^{\circ} \mathrm{C}$ for $5 \mathrm{~min} 40 \mathrm{~s}$ and $72^{\circ} \mathrm{C}$ for $10 \mathrm{~min}$. The 115 amplified fragment was cloned into the pMD19-T for sequencing. The primers had BglII and 116 NotI restriction enzyme sites, thus the fragment was obtained by digestion with these enzyme 117 and then ligated into the ps11180-Hr3-A4-SV40 expression vector (stored in our laboratory).

118 The vectors were named as the BmVgR and BmVgR', respectively. Sf9 cells were transfected 119 with BmVgR/ BmVgR' using Cellfection II (Invitrogen, Carlsbad, CA, USA). All procedures 120 were performed according to the manufacturer's instructions. After refreshing with complete 121 medium and culturing the cells for $72 \mathrm{~h}$, membrane proteins from the cells and ovaries were 122 extracted using strong RIPA lysis (Beyotime Biotechnology, China), and expression of the $123 \mathrm{BmVgR}$ protein was analyzed by western blotting. Transfected cells were also used to 124 determine the localization of $\mathrm{BmVgR}$ by immunofluorescence assays.

\subsection{Insect}

126 Silkworm strain Dazao was obtained from the silkworm genebank at Southwest 
127 University, China. All larvae were reared on fresh mulberry leaves, and pupae were placed at

128 room temperature. Hemolymph and ovary samples were dissected from female silkworms at 129 different stages from wandering to day 5 of pupation and were stored at $-80^{\circ} \mathrm{C}$.

\subsection{Purification and fluorescent labeling of BmVg and BmSP1}

BmSP1 was purified as described previously(Liu et al. 2016). BmVg was purified using

132

a method similar to that used for BmSP1. The hemolymph on day 3 of pupation was diluted in buffer containing $20 \mathrm{mM}$ Tris- $\mathrm{HCl}$ and $100 \mathrm{mM} \mathrm{NaCl}(\mathrm{pH} 7.5)$ and then centrifuged at $10,000 \times \mathrm{g}$ at $4^{\circ} \mathrm{C}$ for $20 \mathrm{~min} . \mathrm{BmVg}$ was precipitated as a stable complex using ammonium sulfate at $50-60 \%$ saturation and was further purified by Q column, followed by HiLoad Superdex S-200 16/600 column purification (GE Healthcare, UK).

The buffer for pure protein was replaced with $0.1 \mathrm{M}$ freshly prepared $\mathrm{NaHCO}_{3}$ buffer ( $\mathrm{pH} 8.3$ ) and diluted to a concentration of $1 \mathrm{mg} / \mathrm{mL}$ measured using the Bradford method (Bradford 1976) before fluorescently labeling. The stock solution of dye was prepared by dissolving $1 \mathrm{mg}$ pHrodo Red dye (Thermo Fisher Scientific, USA) into $150 \mu \mathrm{L}$ dimethyl sulfoxide (DMSO). All procedures were performed according to the manufacturer's instructions. The labeled protein was purified by ultrafiltration. The retentate in the ultrafiltration tube of the last ultrafiltrate was the purified $\mathrm{BmVg}$ labeled with pHrodo-red, while the permeate was collect and used as a control.

\subsection{Co-immunoprecipitation (IP) assay}

The culture medium of BmVgR' expressing in Sf9 cells was tested by western blotting with rabbit anti-BmVgR antibodies. A sample of purified BmVg or BmSP1 from silkworm hemolymph was added into the culture medium and incubated at room temperature for 90 
min to obtain $\mathrm{BmVgR}-\mathrm{BmVg}$ and $\mathrm{BmVgR}-\mathrm{BmSP} 1$ complexs. Next, $8.4 \mu \mathrm{g}$ of rabbit anti-BmVgR antibody diluted in $200 \mu \mathrm{L}$ of culture medium was added to $50 \mu \mathrm{L}$ of $5 \%(\mathrm{w} / \mathrm{v})$ BSA-blocked Dynabeads (Beyotime, China). The Dynabeads were used according to the manufacturer's instructions. In order to exclude interference of non-specifically bound protein, the beads- $\mathrm{Ab}-\mathrm{BmVgR}-\mathrm{BmVg} / \mathrm{BmSP} 1$ complex was washed three times with acidic phosphate-buffered saline (PBS: $137 \mathrm{mM} \mathrm{NaCl}, 2.7 \mathrm{mM} \mathrm{KCl}, 10 \mathrm{mM} \mathrm{Na} 2 \mathrm{HPO} 4$, and 1.8mM $\mathrm{KH} 2 \mathrm{PO} 4 \mathrm{pH}=6.5$ ), and the final wash buffer was evaluated. The complex was resuspended in $100 \mu \mathrm{L}$ of PBS (pH 7.4) and transferred into a clean tube. Next, $20 \mu \mathrm{L}$ of $5 \times$ sodium dodecyl sulfate polyacrylamide gel electrophoresis (SDS-PAGE) loading buffer was added, and the mixture was heated for $10 \mathrm{~min}$ at $100{ }^{\circ} \mathrm{C}$. The eluate from the beads was subjected to SDS-PAGE $(8 \%[\mathrm{w} / \mathrm{v}]$ polyacrylamide gels $)$ and detected by western-blotting with anti-BmVg and anti-BmSP1 antibody.

\subsection{Semi-quantitative reverse transcription ( $R T-P C R)$}

Total RNA was extracted from the ovaries of female silkworms using TRIzol (Thermo Fisher Scientific), and cDNA was generated using M-MLV reverse transcriptase (Promega, Madison, WI, USA). All kits were used according to the manufacturer's instructions. The forward primer for BmSP1 (NM_001113276.2) was 5'-ATTGACTGGCGTAAGGGAG-3', and the reverse primer was 5'-GCTGGGAAGACGGATTTT-3'. PCR was carried out at $94^{\circ} \mathrm{C}$ for $5 \mathrm{~min}$; followed by 25 cycles of $94^{\circ} \mathrm{C}$ for $30 \mathrm{~s}, 53^{\circ} \mathrm{C}$ for $30 \mathrm{~s}$, and $72^{\circ} \mathrm{C}$ for $15 \mathrm{~s}$; and a final extension at $72^{\circ} \mathrm{C}$ for 10 min. BmActin3 was used as a control (forward primer: 5'-AACACCCCGTCCTGCTCACTG-3', and primer: 5'-GGGCGAGACGTGTGATTTCCT-3'). For Bmactin, PCR was carried out at $94^{\circ} \mathrm{C}$ for 5 
171

172

173

174

min; followed by 25 cycles of $94^{\circ} \mathrm{C}$ for $30 \mathrm{~s}, 53^{\circ} \mathrm{C}$ for $30 \mathrm{~s}$, and $72^{\circ} \mathrm{C}$ for $30 \mathrm{~s}$; and a final extension at $72^{\circ} \mathrm{C}$ for $10 \mathrm{~min}$

\subsection{Protein preparation and detection}

Protein samples from total ovaries at different phases were prepared as described (Meng et al. 2009). The proteins were subjected to SDS-PAGE $(10 \%[\mathrm{w} / \mathrm{v}]$ poly-acrylamide gel $)$ according to the method of Laemmli (Laemmli. 1970). The proteins in the gel were transferred electrophoretically onto polyvinylidene difluoride membranes (Roche, Basel, Switzerland) for western blot assay, and imaged using by the ChemiScope instrument.

Hemolymph specimens were treated as previously described. After separation by SDS-PAGE (10\% [w/v] polyacrylamide gels) according to the method of Laemmli, the gel was stained with Coomassie brilliant blue.

\subsection{Immunofluorescence histochemistry}

On day 2 and 5 of pupation, ovaries were dissected from female silkworms, and immunofluorescence histochemistry was conducted as previously described(Shen et al. 2015) using anti-BmSP1, anti-BmVg and anti-BmVgR antibodies (all antibody were prepared in our laboratory).

\subsection{Detection of ligand after incubation with insect cells transfected with BmVgR and} ovaries collected on day 2 of pupation

For endocytic uptake, proteins without fluorescent signals were diluted to an appropriate concentration using HEPES buffer, and Sf9 cells were stably transfected in 6 well cell culture plates (for westen blotting) and 24-well cell culture plates (for immunofluorescence), starved for 30 min with Grace's medium without fetal bovine serum, and incubated with a certain 
concentration of the ligand protein for $60 \mathrm{~min}$ at $27^{\circ} \mathrm{C}$ after incubating at $4^{\circ} \mathrm{C}$ for $20 \mathrm{~min}$.

194 The Sf9 cells were then washed with $0.1 \mathrm{M}$ glycine and $0.1 \mathrm{M} \mathrm{NaCl}(\mathrm{pH} 3.0)$ to remove any

uninternalized ligand (Sun et al. 2005). After confirming that BmVgR was expressed in the transfected cells, ligand uptake was detected by immunofluorescence and western blot analysis using anti-BmSP1 and anti-BmVg antibodies. Each uptake experiment was performed three times.

Ovary tissues were excised from pupa on day 2 of pupation and incubated with pHrodo ${ }^{\mathrm{TM}}$ red-labeled $\mathrm{BmVg}$ at $27^{\circ} \mathrm{C}$ for $1 \mathrm{~h}$. The fluorescence signals of ovaries and ovary ultrathin frozen sections were then observed under a fluorescence microscope (Olympus, Japan).

\section{Results}

\subsection{Expression, localization and in-vitro binding of BmVgR}

To clarify the function of $\mathrm{BmVgR}$, we constructed a $\mathrm{BmVgR}$ cell-expression vector to analyze the function of $\mathrm{BmVgR}$ in $\mathrm{Sf9}$ cells, and another BmVgR' (whole extracellular domain of BmVgR) cell-expression vector to obtain sufficient amounts of protein to perform in-vitro binding assays. The structure schematic of $\mathrm{BmVgR}$ and $\mathrm{BmVgR}$ ' is shown in Fig.1A. BmVgR espressed in Sf9 cells had a molecular weight approximate 250kDa (Fig.1B), consistent with that in the ovary (Fig.1C). BmVgR was located on the outside of the nucleus, i.e., cell membrane and cytoplasm of Sf9 cells (Fig.1D), consistent with its function as a membrane receptor. 
214 LBD1 of BmVgR was shown to interact with BmVg and BmSP1 in a previous study. In 215 our study, the two female-specific and highly expressed protein, BmVg (Fig. 1F) and 216 BmSP1(Fig.1G) were also found to interact with BmVgR in vitro. However, the binding 217 capacity of $\mathrm{BmVg}$ was much weaker than that of BmSP1, which may due to acidic buffer $218(\mathrm{pH}=6.5)$ used to wash the non-specific binding proteins. To the best of our knowledge, the 219 interaction between $\mathrm{BmVg}$ and $\mathrm{BmVgR}$ was dependent on a neutral $\mathrm{pH}$, while the acidic environment promote dissociation of the interaction (Jeon and Blacklow 2003).

\subsection{Uptake of BmVg by Sf9 cells transfected with BmVgR}

Next, we performed uptake assays after confirming that BmVgR can be stably expressed in $\mathrm{Sf} 9$ cells. We also detected and verified the expression of $\mathrm{BmVgR}$ for each endocytosis experiment. After incubating with hemolymph proteins on day 2 of pupation or $1 \mathrm{mg} / \mathrm{mL}$

$\mathrm{BmVg}$, Sf9 cells overexpressing BmVgR could assimilate more BmVg (Fig. 2A). A relatively weak band was observed after incubation with $1 \mathrm{mg} / \mathrm{mL} \mathrm{BmVg}$, partly because $\mathrm{BmVg}$ showed some degradation after storage at a freezing temperature. Subsequently, we re-purified the BmVg protein (Fig. S1-C.D) and confirmed our hypothesis. Immunofluorescence assays further confirmed that $\mathrm{BmVg}$ was absorbed into the $\mathrm{Sf} 9$ cells (Fig. 2B), avoiding false-positive signal caused by BmVg binding to cell surface protein. Thus, Sf9 cells could be used as the appropriate cells to study the uptake of BmVgR. 
cells. Because the state of $\mathrm{BmVg}$ has an impact on absorption by certain cells, we purified BmSP1 from the hemolymph of female silkworms in the wandering stage(Fig. S1-A.B). Compared with the control, the amount of BmSP1 did not increase in Sf9 cells overexpressing BmVgR (Fig. 3A), and even decrease slightly. Additionally, repeated experiments showed that BmSP1 did not associate with BmVgR entering into cells. Sf9 cells with or without BmVgR could assimilate almost the same amount of BmSP1 into Sf9 cells by immunofluorescence (Fig. 3B). Taken together, these results indicated that BmSP1 entered the cells independent of $\mathrm{BmVgR}$. In other words, $\mathrm{BmVgR}$ was not involved in the transport of BmSP1 into Sf9 cells.

\subsection{BmVg was transported into the ovaries by BmVgR through co-localization and} endocytosis

To determine the relationship between $\mathrm{BmVgR}$ and $\mathrm{BmVg}$, ovaries of silkworms on days 2 and 5 of pupation were collected and detected by immunofluorescence. During the early period on day 2 of pupation, $\mathrm{BmVgR}$ was distributed near the oocyte membrane and remained evenly distributed in the oocyte cytoplasm (Fig. 4A), whereas BmVg was mainly localized inside the follicular cell layer and oocyte membrane (Fig. 4C) where BmVgR and $\mathrm{BmVg}$ could interacted with each other. The BmVgR was mainly located near the oocyte membrane (Fig. 4B) on day 5 of pupation, and the ovaries exhibited the most BmVg in the oocyte cytoplasm (Fig. 4D). These data indicated that BmVgR would return to the oocyte membrane after transporting $\mathrm{BmVg}$ into the oocyte cytoplasm.

To further confirm that $\mathrm{BmVg}$ was taken up by the ovaries, we used pHrodo red dye to 
256

label BmVg (Fig. S1-E). pHrodo-red was selected because this dye is $\mathrm{pH}$ sensitive, the fluorescence intensity is stronger as the $\mathrm{pH}$ decreases. To the best of our knowledge, $\mathrm{Vg}$ uptake by VgR depends on vesicles, which eventually fuse with the lysosome, making the environment of $\mathrm{Vg}$ acidic. As a result, the fluorescence intensity of $\mathrm{Vg}$ uptake by the cells was not disrupted by weak interference signals, such as signals from residual dyes in the marked BmVg and extracellular marked BmVg. By SDS-PAGE, the large subunits of BmVg were marked with the red dye. When incubated with the dissociated ovaries on day 2 of pupation, the pHrodo red-labeled $\mathrm{BmVg}$ was observed in oocytes and the interspace. Moreover, the $\mathrm{BmVg}$ was located in the inner region of the oocyte membrane in ovary slices, similar to the location of $\mathrm{BmVg}$ indicated by immunofluorescence on day 2 of pupation (Fig. $4 \mathrm{C})$, supporting that the $\mathrm{BmVg}$ was transported into the ovary.

\subsection{Expression patterns and localization of BmSP1 in the ovaries}

Next, we aimed to clarify the relationship between $\mathrm{BmVgR}$ and BmSP1 in vivo. However, BmVgR was almost undetectable in the fat body when most BmSP1 was absorbed (data not shown). Therefore, we next examined whether BmSP1, which exhibited high levels in the hemolymph, was taken up by the ovaries with high levels of $\mathrm{BmVgR}$ in the oocyte membrane. On P2, BmSP1 was localizd similar to the BmVgR; however, the BmSP1 was very low (Fig. 5A). A certain amount of BmSP1 protein was detected in female ovaries from wandering to day 3 of pupation (Fig. 5B). RT-PCR showed that the level of BmSP1 mRNA in the ovary was higher in wandering and very low in pupae (Fig. 5C), with trends similar to those of BmSP1 protein. BmSP1 existed in the hemolymph from wandering to day 5 of pupation (Fig. 5D). On day 5 of pupation, a large amount of $\mathrm{BmVgR}$ was expressed in the 
ovary and the oocyte membrane (Fig. 4B); however, BmSP1 was not detected in ovary at this phase, suggesting that BmSP1 was completely synthesized in the ovary, or partly absorbed from the hemolymph by an unknown mechanism and receptor. In summary, BmSP1 protein in ovary was also independent of BmVgR.

\section{Discussion}

$\mathrm{Vg}$ is mainly endocytosed by $\mathrm{VgR}$ into the ovaries and the endocytosis mechanism has been studied extensively in mosquitos (Snigirevskaya et al. 1997). In our study, BmVgR was distributed in the oocyte cytoplasm and membrane at the early phase of pupation, indicating that $\mathrm{BmVgR}$ was synthesized in oocytes and finally located in the oocyte membrane. During this phase, $\mathrm{BmVg}$ was localized similar to $\mathrm{BmVgR}$ in the oocyte membrane, suggesting that the two proteins have an interacted with each other. During the late phase of pupation, $\mathrm{BmVgR}$ was mainly distributed in the oocyte membrane, and $\mathrm{BmVg}$ was mainly accumulated in the oocyte cytoplasm, indicating that $\mathrm{BmVgR}$ was eventually returned to the oocyte membrane. Similar localization patterns have been observed during vitellogenesis in Solenopsis invicta Buren and Cutthroat trout(Lu et al. 2009; Mizuta et al. 2013). Vg is synthesized in the female fat body, secreted into the blood and endocytosed by the ovaries (Raikhel and Dhadialla 1992). Moreover, Vg localizes with VgR during the early stage of vitellogenesis and later deposits in the yolk granules. In our study, we acquired similar localization using immunofluorescence and endocytosis of pHrodo red labeled BmVg. Sf9 cells, derived from Spodoptera frugiperda ovaries and do not express endogenous BmVgR and $\mathrm{BmVg}$, were selected as the ideal cell. Sf9 cells could absorb more BmVg when it was over-expressed with BmVgR. 
In silkworms, the uptake of storage proteins by insect fat body cells exhibits several unique features, and it is still unclear whether this process conforms to the standard scheme of eukaryotic endocytosis (Burmester and Scheller 1999). Recently, the receptor responsible for storage protein was identified in Sarcophaga peregrine (Ueno and Natori 1984);

Calliphora vicina (Burmester and Scheller 1997); Drosophila melanogaster (Burmester et al. 1999); Corcyra cephalonica (Damara et al. 2010) and Helicoverpa zea (Wang 1994b). In

silkworms, the receptor for BmSP1 is still unclear. In contrast, BmVgR is known to bind 
322 323 study.

\section{Acknowledgements}

325

\section{References} 10.1002/cbic. 200300580 . 248-254.

transported by $\mathrm{BmVgR}$ though a mechanism similar to that of $\mathrm{BmVg}$, as found in this

We thank Dr. Y. Hou for kindly providing the anti-SP1 antibody. This work was supported by the State Key Program of National Science of China (no.31530071), National Natural Science Foundation of China (no.31402139 and no:31401048).

\section{Competing financial interests}

We declare we have no competing interests.

Amdam, G.V., Norberg, K., Hagen, A., and Omholt, S.W. 2003. Social exploitation of vitellogenin. Proceedings of the National Academy of Sciences of the United States of America 100(4): 1799-1802. doi: 10.1073/pnas.0333979100.

Andersen, O.M., and Petersen, H.H. 2003. New light on a long-known protein family. Chembiochem : a European journal of chemical biology 4(11): 1137-1146. doi:

Bradford, M.M. 1976. A rapid and sensitive method for the quantitation of microgram quantities of protein utilizing the principle of protein-dye binding. Analytical biochemistry 72:

Bujo, H., Hermann, M., Kaderli, M.O., Jacobsen, L., Sugawara, S., Nimpf, J., Yamamoto, T., and Schneider, W.J. 1994. Chicken oocyte growth is mediated by an eight ligand binding repeat member of the LDL receptor family. The EMBO journal 13(21): 5165-5175.

Burmester, T., Antoniewski, C., and Lepesant, J.A. 1999. Ecdysone-regulation of synthesis 
344 and processing of fat body protein 1, the larval serum protein receptor of Drosophila 345 melanogaster. Eur J Biochem 262(1): 49-55.

346 Burmester, T., and Scheller, K. 1997. Developmentally controlled cleavage of the Calliphora 347 arylphorin receptor and posttranslational action of the steroid hormone 20-hydroxyecdysone. 348 Eur J Biochem 247(2): 695-702.

349 Burmester, T., and Scheller, K. 1999. Ligands and receptors: common theme in insect storage 350 protein transport. Die Naturwissenschaften 86(10): 468-474.

351 Ciudad, L., Piulachs, M.D., and Belles, X. 2006. Systemic RNAi of the cockroach 352 vitellogenin receptor results in a phenotype similar to that of the Drosophila yolkless mutant. 353 The FEBS journal 273(2): 325-335. doi: 10.1111/j.1742-4658.2005.05066.x.

354 Damara, M., Gullipalli, D., and Dutta-Gupta, A. 2010. Cloning and expression of fat body 355 hexamerin receptor and its identification in other hexamerin sequestering tissue of rice moth, 356 Corcyra cephalonica. J Insect Physiol 56(9): 1071-1077. doi: 10.1016/j.jinsphys.2010.02.017.

357 Gliemann, J. 1998. Receptors of the low density lipoprotein (LDL) receptor family in man. 358 Multiple functions of the large family members via interaction with complex ligands. 359 Biological chemistry 379(8-9): 951-964.

360 Goldstein, J.L., Brown, M.S., Anderson, R.G., Russell, D.W., and Schneider, W.J. 1985. 361 Receptor-mediated endocytosis: concepts emerging from the LDL receptor system. Annual 362 review of cell biology 1: 1-39. doi: 10.1146/annurev.cb.01.110185.000245.

363 Hiesberger, T., Hermann, M., Jacobsen, L., Novak, S., Hodits, R.A., Bujo, H., Meilinger, M., 364 Huttinger, M., Schneider, W.J., and Nimpf, J. 1995. The Chicken Oocyte Receptor for Yolk 365 Precursors as a Model for Studying the Action of Receptor-Associated Protein and 
366 Lactoferrin. Journal of Biological Chemistry 270(31): 18219-18226.

367 Hussain, M.M., Strickland, D.K., and Bakillah, A. 1999. The mammalian low-density 368 lipoprotein receptor family. Annual review of nutrition 19: 141-172. doi: 369 10.1146/annurev.nutr.19.1.141.

370 Jacobsen, L., Hermann, M., Vieira, P.M., Schneider, W.J., and Nimpf, J. 1995. The chicken 371 oocyte receptor for lipoprotein deposition recognizes alpha 2-macroglobulin. The Journal of 372 biological chemistry 270(12): 6468-6475.

373 Jeon, H., and Blacklow, S.C. 2003. An intramolecular spin of the LDL receptor beta propeller. 374 Structure (London, England : 1993) 11(2): 133-136.

375 Laemmli, U.K. 1970. Cleavage of structural proteins during the assembly of the head of 376 bacteriophage T4. Nature 227(5259): 680-685.

377 Lin, W.J., Chien, C.Y., Tsai, C.L., and Chen, M.E. 2015. A NONOVARY-SPECIFIC 378 VITELLOGENIN RECEPTOR FROM THE ORIENTAL FRUIT FLY, Bactrocera dorsalis 379 (HENDEL). Archives of insect biochemistry and physiology 90(4): 169-180. doi: $380 \quad 10.1002 / \operatorname{arch} .21252$.

Lin, Y., Meng, Y., Wang, Y.X., Luo, J., Katsuma, S., Yang, C.W., Banno, Y., Kusakabe, T., 382

Shimada, T., and Xia, Q.Y. 2013. Vitellogenin receptor mutation leads to the oogenesis mutant phenotype "scanty vitellin" of the silkworm, Bombyx mori. The Journal of biological chemistry 288(19): 13345-13355. doi: 10.1074/jbc.M113.462556.

Liu, L., Wang, Y., Li, Y., Lin, Y., Hou, Y., Zhang, Y., Wei, S., Zhao, P., and He, H. 2016. 
388 Lu, H.L., Vinson, S.B., and Pietrantonio, P.V. 2009. Oocyte membrane localization of 389 vitellogenin receptor coincides with queen flying age, and receptor silencing by RNAi 390 disrupts egg formation in fire ant virgin queens. The FEBS journal 276(11): 3110-3123. doi: 10.1111/j.1742-4658.2009.07029.x.

Meng, Y., Katsuma, S., Daimon, T., Banno, Y., Uchino, K., Sezutsu, H., Tamura, T., Mita, K., and Shimada, T. 2009. The silkworm mutant lemon (lemon lethal) is a potential insect model 11698-11705. doi: 10.1074/jbc.M900485200. 
410 Sakurai, H., Fujii, T., Izumi, S., and Tomino, S. 1988. Structure and expression of gene

411 coding for sex-specific storage protein of Bombyx mori. The Journal of biological chemistry

$412 \quad$ 263(16): 7876-7880.

413 Sappington, T.W., Kokoza, V.A., Cho, W.L., and Raikhel, A.S. 1996. Molecular 414 characterization of the mosquito vitellogenin receptor reveals unexpected high homology to 415 the Drosophila yolk protein receptor. Proceedings of the National Academy of Sciences of the 416 United States of America 93(17): 8934-8939.

417 Sappington, T.W., and Raikhel, A.S. 1998. Molecular characteristics of insect vitellogenins 418 and vitellogenin receptors. Insect Biochem Molec 28(5-6): 277-300. doi: Doi 10.1016/S0965-1748(97)00110-0.

420 Schjeide, O.A., Briles, W.E., Holshouser, S., and Jones, D.G. 1976. Effect of "restricted 421 ovulator" gene on uptake of yolk-precursor protein. Cell Tissue Res 166(1): 109-116.

422 Schonbaum, C.P., Lee, S., and Mahowald, A.P. 1995. The Drosophila yolkless gene encodes a 423 vitellogenin receptor belonging to the low density lipoprotein receptor superfamily. 424 Proceedings of the National Academy of Sciences of the United States of America 92(5): $425 \quad 1485-1489$.

426 Shen, G., Lin, Y., Yang, C., Xing, R., Zhang, H., Chen, E., Han, C., Liu, H., Zhang, W., and $427 \mathrm{Xia}$, Q. 2015. Vertebrate estrogen regulates the development of female characteristics in 428 silkworm, Bombyx mori. General and comparative endocrinology 210: 30-37. doi: 10.1016/j.ygcen.2014.09.016.

430 Shu, Y.H., Wang, J.W., Lu, K., Zhou, J.L., Zhou, Q., and Zhang, G.R. 2011. The first 431 vitellogenin receptor from a Lepidopteran insect: molecular characterization, expression 
432 patterns and RNA interference analysis. Insect molecular biology 20(1): 61-73. doi: $433 \quad 10.1111 /$ j.1365-2583.2010.01054.x.

434 Smith, A.D., and Reuben Kaufman, W. 2013. Molecular characterization of the vitellogenin 435 receptor from the tick, Amblyomma hebraeum (Acari: Ixodidae). Insect Biochem Mol Biol 436 $10.1007 / \mathrm{s} 004410050919$.

440 Sun, X.J., Yau, V.K., Briggs, B.J., and Whittaker, G.R. 2005. Role of clathrin-mediated 10.1016/j.virol.2005.05.006.

Tufail, M., and Takeda, M. 2007. Molecular cloning and developmental expression pattern of 37(3): 235-245. doi: 10.1016/j.ibmb.2006.11.007. 30K proteins. Insect Biochem Mol Biol 42(11): 827-834. 
454 Zhong, R., Ding, T.B., Niu, J.Z., Xia, W.K., Liao, C.Y., Dou, W., and Wang, J.J. 2015.

455 Molecular characterization of vitellogenin and its receptor genes from citrus red mite,

456 Panonychus citri (McGregor). International journal of molecular sciences 16(3): 4759-4773.

457 doi: 10.3390/ijms16034759.

\section{Figure Legends}

Fig.1. Expression, localization and in-vitro binding of BmVgR and BmVgR'. (A)

Structural schematic of BmVgR and BmVgR'. S, signal peptide; LBD, ligand-binding domain;

EGFP, epidermal growth factor precursor homology domain ; O, O-linked sugar domain; T,

transmembrane domain; C, cytoplasmic domain. (B) Proteins extracted from Sf9 cells were detected using anti-BmVgR antibodies. BmVgR and Con indicated the Sf9 cells transfected with BmVgR over-expression and control vector. (C) Proteins of silkworm ovaries on P5 were detected with anti-BmVgR antibodies. (D) The localization of BmVgR in Sf9 cells was detected by immunofluorescence. FITC, localization of BmVgR; MERGE, combined figures of cell nuclei (DAPI) and FITC. (E) Overexpressed of BmVgR' protein in Sf9 cells. (F) BmVgR' interacted with $\mathrm{BmVg}$, as determined by co-IP. (G) BmVgR' interacted with BmSP1, as determined by co-IP.

Fig.2. Uptake of BmVg by Sf9 cells with and without BmVgR. (A) BmVg was absorbed by Sf9 cells after incubating with hemolymph on day 2 of pupation (P2-hem) or $1 \mathrm{mg} / \mathrm{mL}$ $\mathrm{BmVg}(\mathrm{BmVg})$, and western blotting was used for detection. Tubulin was used as the internal reference. The right figure in (A) shows $\mathrm{BmVg}$ protein levels reflected by the relative gray 475 levels of left figure in (A) measured by Image J. Experiments were repeated three times 
476

independently, and the relative gray level is shown as the mean $\pm \mathrm{SD} ; * * \mathrm{P}<0.01$. (B) $\mathrm{BmVg}$ taken up by Sf9 cells was detected by immunofluorescence. FITC staining indicated the the localization of $\mathrm{BmVg}$, and the MERGE image showed the combination of FITC and DAPI staining.

Fig.3. Uptake of BmSP1 by Sf9 cells with and without BmVgR. (A) BmSP1 uptake by Sf9 cells was detected by western blotting, and tubulin was used as the internal reference. The last two lanes show the corresponding cell culture medium. The right figure in (B) is the BmSP1 protein level, which reflects the relative gray level of the first two lanes in left figure in (A), as measured by Image J. Experiments were repeated three times independently, and the relative gray level is given as the means $\pm \mathrm{SD}$. (B) BmSP1 taken up by Sf9 cells was detected by immunofluorescence. FITC, signal of BmSP1; MERGE image, the combination of FITC and DAPI staining.

\section{Fig.4. Immunofluorescence images of vitellogenin receptor(VgR) and vitellogenin( $\mathrm{Vg})$ in} silkworm ovaries on $\mathrm{P} 2$ and $\mathrm{P5}$, and pHrodo $^{\mathrm{TM}}$ red-labeled BmVg uptake by oocyte on P2. (A) The localization of BmVgR on P2. (B) The localization of BmVgR on P5. The white arrows indicate the nuclei of follicular cells, which are localized outside the oocyte, while red arrows indicate the localization of $\mathrm{VgR}$ in the oocyte membrane, showing the location of the oocyte membrane. (C) The localization of BmVg on P2. (D) The localization of BmVg on P5. (E) The localization of pHrodo red-labeled BmVg in the ovary and 8 - $\mu \mathrm{m}$-thick sections of ovary on P2 after incubation with $200 \mu \mathrm{g} / \mathrm{mL}$ pHrodo red-labeled BmVg. P2, P5; 2, 5 days of pupation. W-ovary, R-ovary, R-ovary-slice, the bright field image of ovaries, figures using the red fluorescence excitation light channel to observe ovaries and ovary-slice. Control and 
498

499

BmVg-Red indicated the permeate and retentate (purified BmVg labeled with phrodo-red) in an ultrafiltrate tube for the last ultrafiltrate, respectively.

Fig.5. Localization and expression patterns of BmSP1 in the ovary. (A) Localization of BmSP1 in silkworm ovaries on P2 was analyzed by immunofluorescence. (B) BmSP1 protein in the ovaries duing different phases. Tubulin was used as internal reference, with $40 \mu \mathrm{g}$ of total protein in each lane. (C) Gene expression pattern of BmSP1 in the ovary, as detected by RT-PCR. (D) BmSP1 in the hemolymph was analyzed by SDS-PAGE, with $40 \mu \mathrm{g}$ total protein in each lane. (L507, day 7 of the fifth instar larvae; W1, W2, and W3: days 1, 2, and 3 of wandering; P1, P3, and P5: days 1, 3, and 5 of pupation, respectively).

Fig.S1. Purification and fluorescent labeling of BmVg and BmSP1. (A) The UV-280 nm curve (indicating the BmSP1 protein content) of the HiLoad Superdex S-200 16/600 column used to purify the BmSP1 protein. (B) The corresponding purified BmSP1 protein collected from the peak on the curve was detected by Coomassie blue staining. (C) The UV-280 nm curve of the HiLoad Superdex S-200 16/600 column used to purify the BmVg protein. (D). The corresponding purified $\mathrm{BmVg}$ protein collected from the peak on the curve was detected by Coomassie blue staining. (E) The $\mathrm{pHrodo}^{\mathrm{TM}}$ red-labeled $\mathrm{BmVg}$ protein was detected directly by SDS-PAGE and the subsequent Coomassie blue staining. 
Fig. 1

A

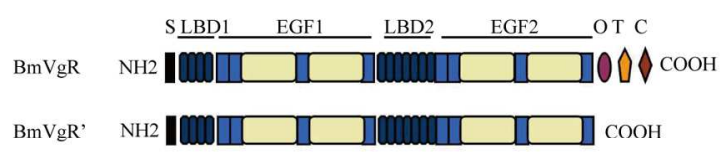

B

C

D FITC

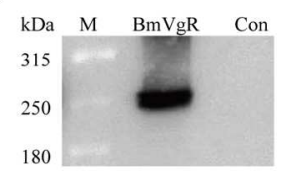

E

F

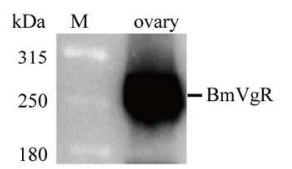

G

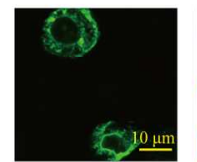

MERGE

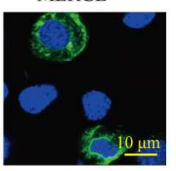

$\begin{array}{rllll}\mathrm{BmSP} 1: & + & - & + \\ \mathrm{BmVgR}: & + & - & + & -\end{array}$

$\begin{array}{rllll}\text { BmVg: } & + & - & + & + \\ \text { BmVgR': } & + & - & + & -\end{array}$

Con: - $-\quad-+$

$\begin{array}{rllll}\text { Anti-BmVgR: } & - & - & + & + \\ \text { Protein G: } & - & - & + & +\end{array}$

Con: $-\quad-+$

Anti-BmVgR: - -++

Protein G: $\quad-++$

kDa Con BmVgR
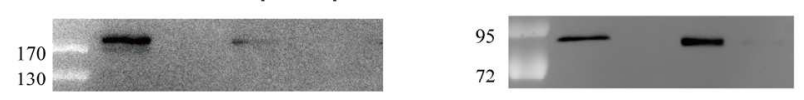

$160 \times 180 \mathrm{~mm}(300 \times 300 \mathrm{DPI})$ 
Fig. 2

A
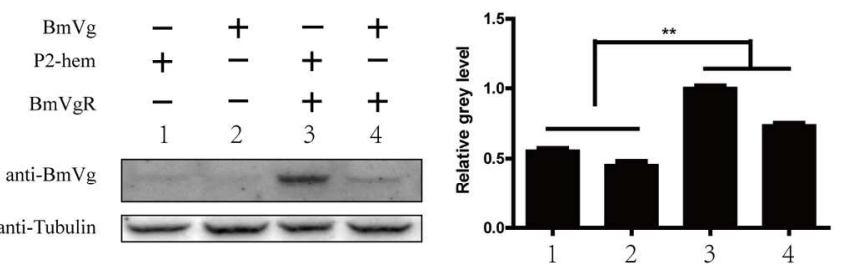

B

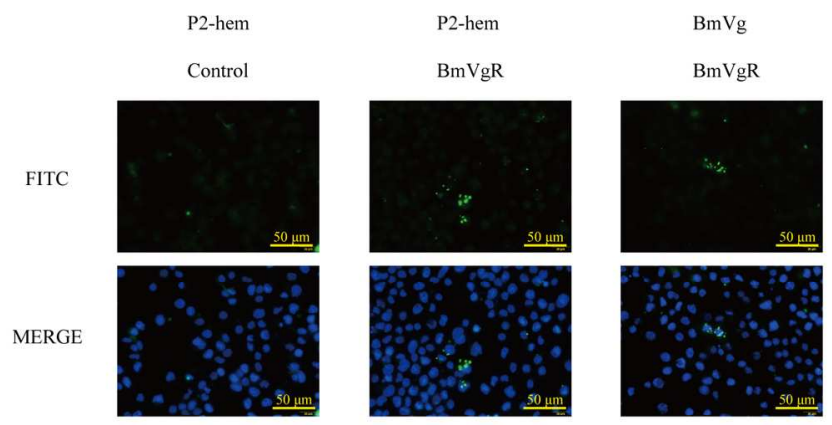

$160 \times 180 \mathrm{~mm}(300 \times 300 \mathrm{DPI})$ 
Fig. 3

A

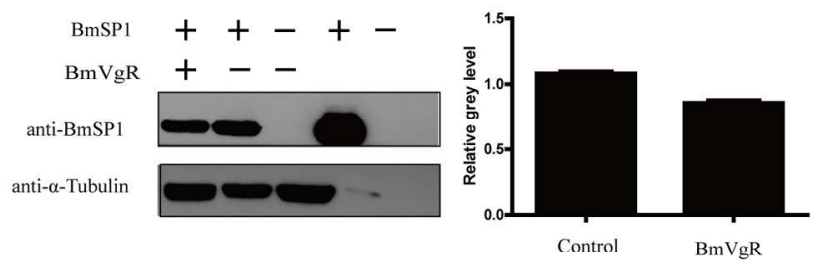

B

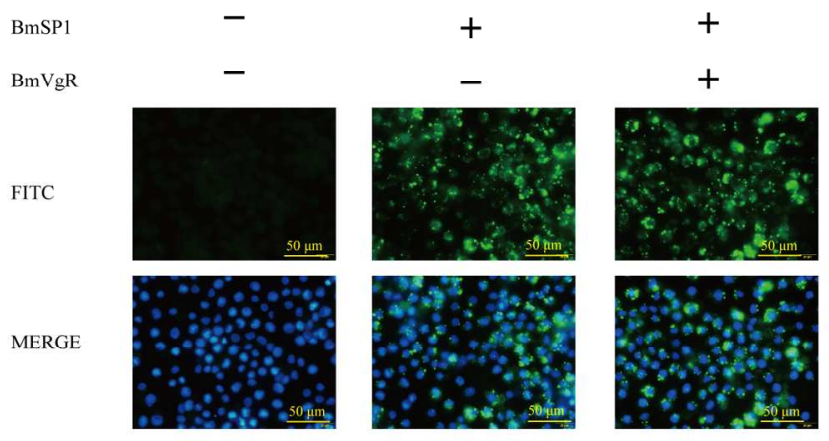

$160 \times 180 \mathrm{~mm}(300 \times 300 \mathrm{DPI})$ 
Fig. 4

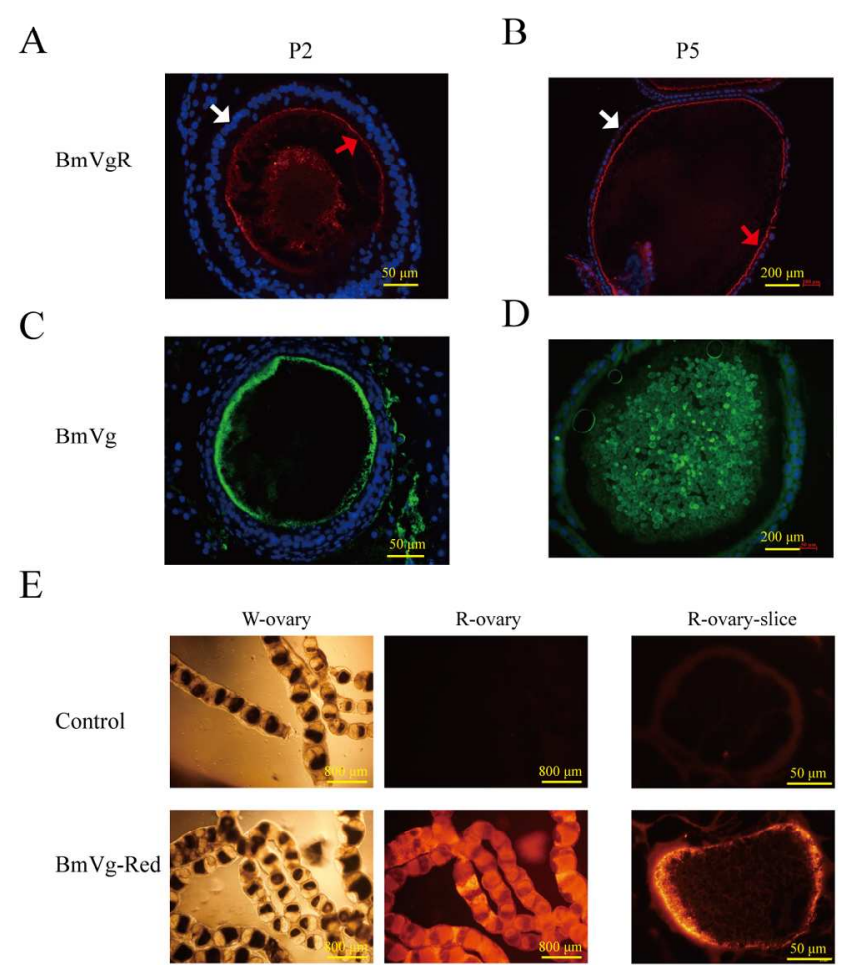

$160 \times 180 \mathrm{~mm}(300 \times 300 \mathrm{DPI})$ 


\section{Fig.5}

A

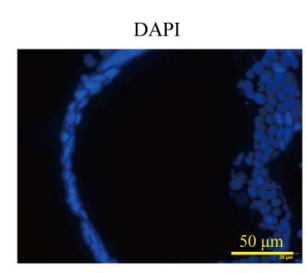

B

anti-BmSP1

anti- $\alpha$-Tubulin

C
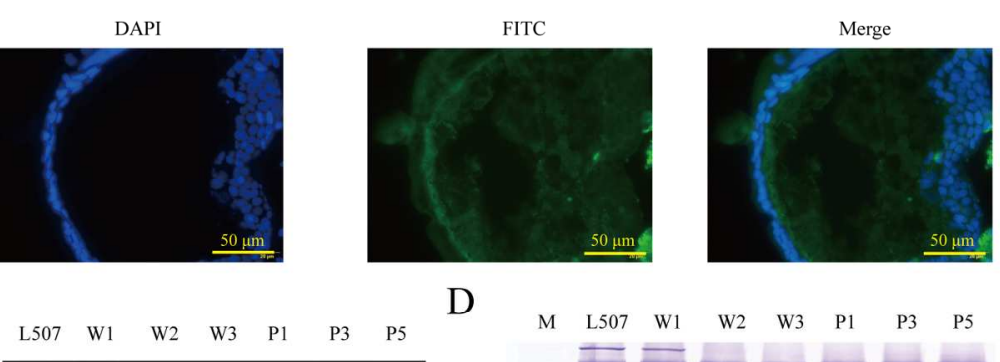

D
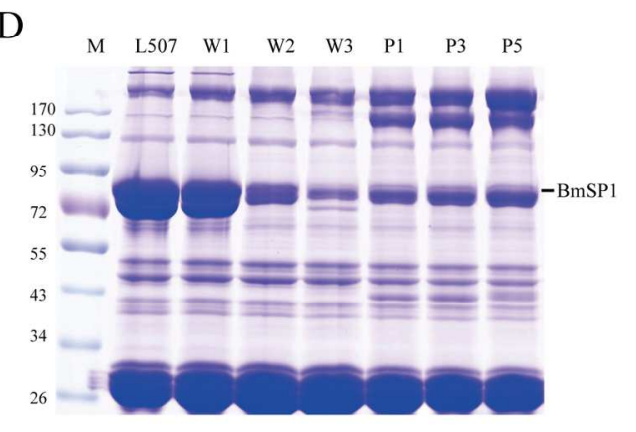

$160 \times 180 \mathrm{~mm}(300 \times 300 \mathrm{DPI})$ 